\title{
Cleated Print Surface for Fused Deposition Modeling
}

\author{
Christopher Scott Shafer ${ }^{1,2}$, Derek Holden Siddel ${ }^{1,2}$ and Amy McDow Elliott ${ }^{1}$ \\ 1. Oak Ridge National Laboratory, Oak Ridge, 37831, United States of America \\ 2. Department of Mechanical Engineering, University of Tennessee Knoxville, Knoxville, 37996, United States of America
}

\begin{abstract}
FDM (fused deposition modeling) has become popular among Additive Manufacturing technologies due to its speed, geometric scalability, and low cost; however, the primitive nature of the FDM build surface fundamentally limits the utility of FDM in terms of reliability, autonomy, and material selection. Currently, FDM relies on adhesive forces between the first layer of a print and the build surface; depending on the materials involved, this adhesive bond may or may not be reliable. Thermal contraction between the build plate and build materials can break that bond, which causes warpage and delamination of the part from the build surface and subsequent failure of the part. Furthermore, with each print, the user must use tools or manual maneuvering to separate the printed part from the build surface as well as retexture or replace the used build surface. In this paper, we present a novel build platform that allows for a mechanical bond between the print and build surface by using dovetail-shaped features. The first layer of the print flows into the features and becomes mechanically captivated by the build platform. Once the print is completed, the platform is rolled or flexed open to release the part from the mechanical bond. This design not only lowers the risk of delamination during printing but also eliminates the need for a user to reset or replace the build surface between print jobs. The effectiveness of each geometry was determined by measuring the distance at the pinch point compared to the distance that the extrusion filled below the pinch point. The captivation ratio was measured to compare the different geometries tested and determine which direction of extrusion creates a better ratio.
\end{abstract}

Key words: Build surface, print surface, fused deposition modeling (FDM), additive manufacturing (AM).

\section{Introduction}

Over the course of development of FDM (fused deposition modeling) AM (additive manufacturing), many improvements have been made to allow for a wide range of materials to be printed. The advancements that have been made to print surfaces, however, are each geared toward specific materials. Considering the narrow range of print surfaces currently developed, it is unknown whether or not other materials may be compatible with a given surface. This leads to one major limitation all FDM printers have, which is that materials can only be successfully printed if they are compatible with the build surface [1]. Furthermore, build surfaces must be carefully monitored and maintained to ensure sufficient sticking during printing, which constitutes a

Corresponding author: Christopher Scott Shafer, Undergraduate Researcher, research fields: mechanical engineering, powder metallurgy, binder jetting additive manufacturing, additive manufacturing systems. significant operator burden. In order to adhere prints to the print bed, many of the build surfaces that are commercially available involve tapes, such as BuildTak $^{\mathrm{TM}}$, PEI sheets, Kapton ${ }^{\circledR}$ tape, and blue painter's tape [2]. Others involve glues, such as CubeStick $^{\mathrm{TM}}$, and WolfBite ${ }^{\mathrm{TM}}$. Unfortunately, large-and small-scale FDM printers still experience delamination issues due to thermal changes, residual stresses, and raster orientation [3-5]. A build platform that is compatible with all FDM materials, requires no replacing or resetting by the operator, and does not rely on adhesive bonding is needed to solve this problem.

The initial bond of the first print layer of an FDM print to the build surface is critical in achieving a successful print. In this work, we sought to design a build platform that is indefinitely reusable and will work with virtually any material by creating a mechanical bond between the print and a dovetail-shaped, cleated build surface. With this 
design, the extrusion of the first printed layer flows into the grooves, mechanically bonding itself to the platform. There are currently no other systems on the market for this type of application; the majority instead rely on adhesive bonding. The United States Patent pending 14/548,701 includes this and any other technologies that utilize "recessive and protrusive" areas to captivate the printed parts [3]. The designs were constructed to create recessive areas into which the extrusion can flow and be captivated. The bond can only be released by bending the platform away from the part to open the grooves, as shown in Fig. 1.

This figure demonstrates how the build surface can be flexed to release the printed part. Another design could be that each row is similar to links in a chain that can be rotated to release the printed part.

\section{Experimental}

To test the effectiveness in captivating the first layer of an FDM print, cleated build surfaces of varying geometries were printed on a Fortus $900 \mathrm{mc}$ FDM printer out of ULTEM 9085; this material was chosen for its toughness and resistance to heat. The cleated platform geometry is comprised of linear rows of "cleats," which are raised features that produce overhangs to capture the extruded plastic from the first layer of the FDM print. The initial design was of a straight-edged trapezoid profile, but parts were difficult to release. A design change was made in which the back corner was rounded. The cleat geometries tested were similar to a dove-tail groove geometry, except that the back edge of each cleat was rounded. This shape allows the cleats to rotate and release the printed part. The depth of the cavity between rows was approximately $0.5^{\prime \prime}$ deep, into which the extrusion would flow. These shapes were arranged in repeating rows in which all of the rounded edges were facing the same direction. Fig. 2 shows a side view of two cleat profiles chosen for this study. One cleat profile is a simple, one-sided dovetail groove with a varying angle (Fig. 2a) and the other is a "wave" profile (Fig. 2b).

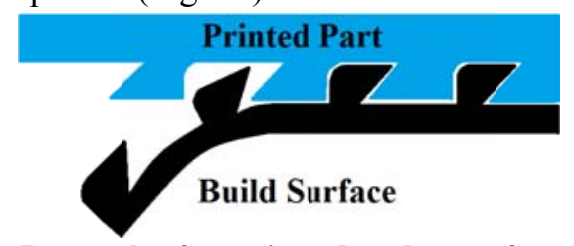

Fig. 1 Removal of captivated polymer from cleated platform.
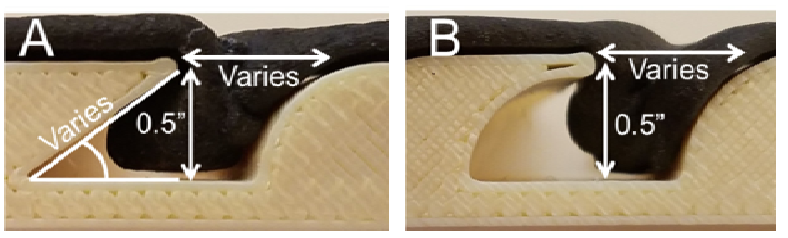

Fig. 2 Cleat Surfaces printed via FDM (tan) in (A) angled and (B) wave profiles.

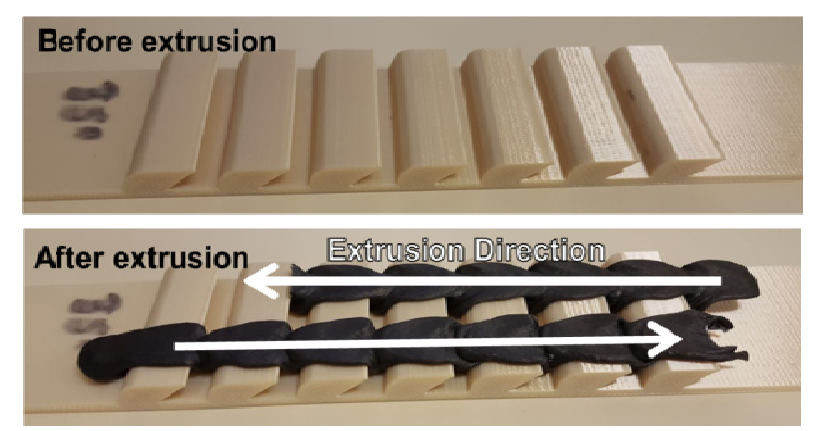

Fig. 3 Cleat geometries (tan) before and after polymer extrusion (black) with the BAAM system.

The overall dimensions of the cleated surfaces were roughly $3 "$ wide and 8 " long, depending on the spacing between rows of cleats. Each printed cleat surface was affixed to the build plate of the BAAM (big area additive manufacturing), FDM printer and Carbon Fiber-doped ABS (Acrylonitrile Butadiene Styrene) plastic was extruded over each surface. The molten ABS was printed in both directions on the build surface in a "U" shape and flowed into the grooves in two different directions, as shown in Fig. 3.

The arrows indicate the direction of extrusion on the cleat surfaces.

During extrusion with the BAAM, the molten plastic flows between the cleats, creates a foot-like profile, and solidifies upon cooling. These feet are what provide the mechanical bond to the print surface (Fig. 4). The critical features of the captivation are the 
width of the foot and the pinch point provided by the cleat profile. As the foot width exceeds the cleat pinch point, the extrusion becomes captivated.

The lightly colored section of the image is the cleated build surface and the dark section is the extrusion. The pinch point can be seen between the rounded back of one row and the knife-edge of the adjacent cleat. The area below the pinch point is where the mechanical bond is formed. The "Foot Width" distance was used for ratio measurements.

Multiple angles of the dovetail profile were tested for BAAM extrusion captivation, including $35^{\circ}, 45^{\circ}$, and $55^{\circ}$. Two wave shapes were also tested with different spacings between rows. The cleat profiles, their dimensions, and the results of extrusion with the BAAM are provided in Table 1.

After extruding over each cleated build surface with the BAAM system, the solidified extrusion was removed by flexing the cleated substrate and it was then measured with calipers. The size of the cleats and BAAM extrusion made measurement trouble-free. After measuring the pinch point length of the cleated profile and the foot widths of each extrusion from each extrusion direction, the measurements were compiled. The captivation ratio for each cleat and extrusion direction was calculated by dividing the foot width by the pinch point in each cleat. An example calculation is as follows: if the pinch point at the top of the trough was measured to be 0.5 " and the extrusion was measured to be 1.0", an extrusion-to-pinch point ratio would result in $1.0 \% / 0.5 \%$ which equals 2.0. An average of three feet of the extrusion was used in the ratio calculation for both directions of extrusion. The ratio data were compiled and displayed in a graph depicting the ratios of with and against the direction of the build surface for all of the variations, as shown in Fig. 4.

\section{Results and Discussion}

The calculated captivation ratios were plotted against the pinch point widths, as shown in Fig. 5. A trend can be seen in the plot between the different geometries that suggests that the captivation ratio decreases with an increase in pinch point width. Although the groove angle also decreases for two of these data points, comparing the two data points for

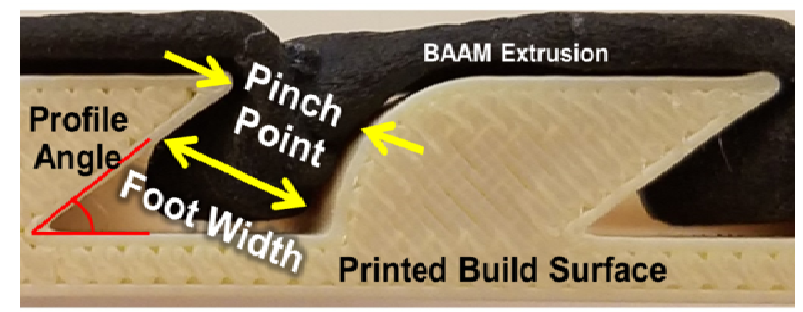

Fig. 4 Side view of cleat profile with captivated polymer extrusion.

Table 1 Cleat geometries (tan) with polymer extruded (black) with- vs. against-extrusion direction. This figure shows all of the geometries as printed on with the extrusion direction indicated. In most cases, it is easily seen that there is a larger foot in the with-direction than the against-direction.

Geometry
$35^{\circ}$ Angle, 0.225" Pinch point
45 Angle, 0.30" Pinch
W50 Angle, 0.38" Pinch
$0.25^{\circ}$ Pinch




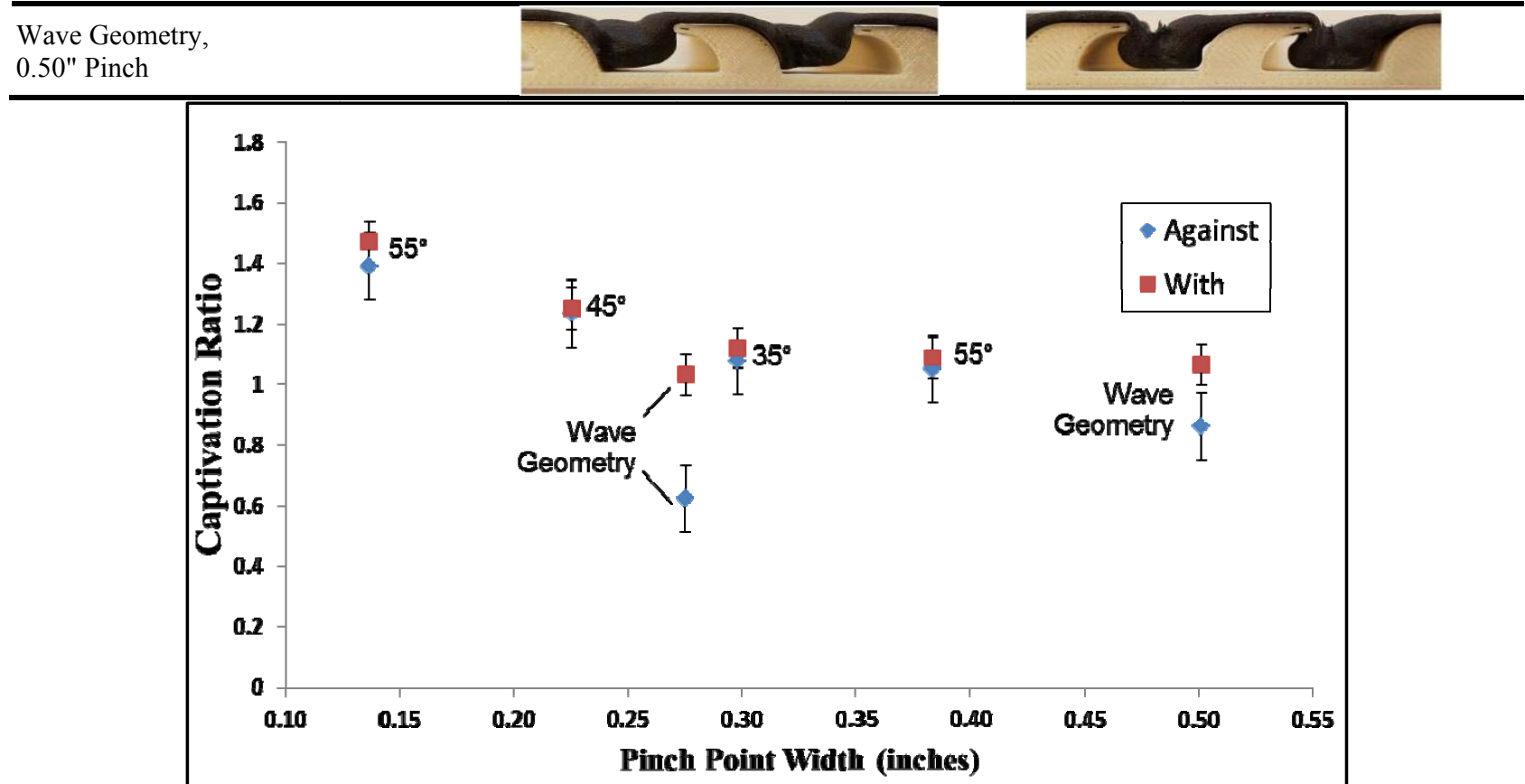

Fig. 5 Captivation ratio vs. extrusion profiles plot.

the 55-degree groove angle at low and high values for pinch point further supports this trend. Additional research is necessary to isolate the groove angle as a variable to discover its effect on captivation. Further, an investigation into the relationship between the nozzle size and/or extrusion rate with the pinch point width is needed to determine the impact of these variables on the captivation ratio.

This graph shows that there was a greater extrusion width in all of the build surfaces when extruded in the with-direction toward the rounded back first. The straight-angled designs all fared better than the wave-shaped designs because the wave shape has a greater directional dependence than the straight-angle designs.

Other trends from the plot include better captivation in extrusions that were deposited with the cleat profiles rather than against. This trend is significantly more pronounced for the wave profiles. When extruding in the direction of the cleat profile, the bead curls under the edge and is pushed farther down as the nozzle passes over, as indicated in Fig. 6 by the curved white arrow. Conversely, in extruding against the cleat profile, the extrusion starts from the bottom of the cavity and is cut off by the overhang feature before it can push the plastic underneath it. Fig. 6 illustrates the difference in flow behavior of the extruded polymer when extruded with and against the cleat profile.

Section $(a, b, c)$ demonstrates the flow of plastic exiting the nozzle in the with-direction; section $(\mathrm{d}, \mathrm{e}, \mathrm{f})$ demonstrates the against-direction. It was hypothesized and proven that the with-direction would roll more plastic underneath the pinch point than the against-direction due to the currents exiting the nozzle.

\section{Conclusions and Future Work}

Captivation of FDM prints by the build surface during printing is a significant challenge with current systems and can lead to delamination and print failure. Further, various materials cannot be printed on any one build surface. However, this cleated build surface provides a solution to those problems. Though additional research is still necessary, it is expected that this build surface functions with any extruded material. It has also proven itself capable of easily captivating and releasing extruded material without the complications 
of print failure or delamination during printing. In this work, cleated build surface geometries were fabricated and tested for their ability to captivate a molten

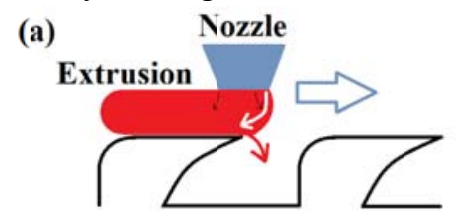

(b)

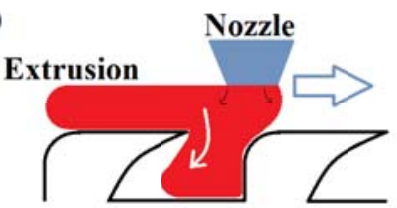

(c)

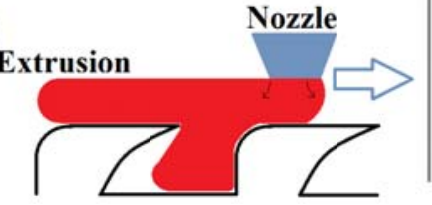

extrusion of thermoplastic as produced in the first

(d)

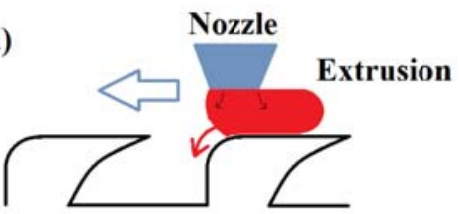

(e)

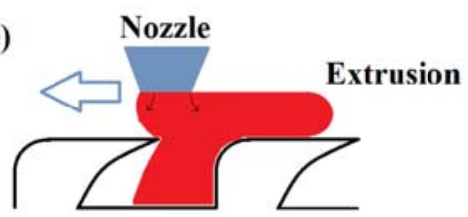

(f) Nozzle

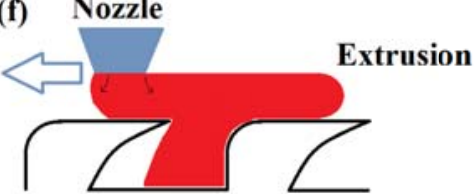

Fig. 6 Flow of molten polymer between cleats during deposition.

layer of an FDM print. The cleat profiles were printed via FDM on a large scale and tested for captivation by the BAAM polymer FDM system. A captivation ratio was calculated based on measurements of the cooled extrusion and the cleat dimensions. The captivation ratio was plotted versus the cleat dimensions and a general trend was seen between the captivation of the extrusion and the pinch point width of the cleats.

Further work is necessary to explore the effects of cleat geometry. Different designs should be researched in order to optimize the system. The extrusion rate should also be taken into consideration as a variable when determining the captivation of extruded polymer. Also, future work will consist of pull tests between printed parts and the build surface to more accurately characterize the captivation of extruded polymer in the cleats. Overall, a cleated build platform would provide a reusable and reliable build surface for FDM printers that would theoretically be universal to all extruded material types. This work is protected by United States Patent pending 14/548,701.

\section{Acknowledgements}

The authors would like to gratefully acknowledge
Brian Post, ORNL for granting access to the BAAM system that made these experiments possible. This material is based upon work supported by the U.S. Department of Energy, Office of Science, Office of Advanced Manufacturing under contract number DE-AC05-00OR22725.

\section{References}

[1] Anderson, T. 2016. "Choosing the Best 3D Printing bed Surface." MatterHackers Inc. Accessed Oct. 7, 2016. http://www.matterhackers.com/news/choosing-the-right-3 d-print-bed-surface.

[2] 3D Printer Plates. 2017. PRINT in Z.com.

[3] Letcher, T., Rankouhi, B., and Javadpour, S. 2015. "Experimental Study of Mechanical Properties of Additively Manufactured ABS Plastic as a Function of Layer Parameters." In Proceedings of the ASME Int. Mech. Eng. Cong. and Exp., p. V02AT02A018-V02AT02A018.

[4] MakerBot Replicator 2X Troubleshooting Maintenance. Brooklyn, NY: MakerBot (2013).

[5] Zhang, Y., and Chou, Y. K. 2006. "Three-Dimensional Finite Element Analysis Simulations of the Fused Deposition Modelling Process." IMechE Vol. 220 Part B, DOI: 10.1243/09544054JEM572.

[6] Elliott, A. M. 2014. Build Platform that Provides Mechanical Engagement with Additive Manufacturing prints. UT-Battelle LLC, assignee. Patent US14548701. 20 Nov. 2014. Print. 